\title{
ESTADO NUTRICIONAL E FATORES ASSOCIADOS AO DÉFICIT DE ESTATURA EM CRIANÇAS ATENDIDAS POR UMA UNIDADE DE ENSINO BÁSICO DE TEMPO INTEGRAL
}

\section{NUTRICIONAL STATUS AND ASSOCIATED FACTORS OF LOW STATURE IN CHILDREN ATTENDED BY A REGULAR SCHOOL OF INTEGRAL TIME}

\author{
Sabrynna Orlonski ${ }^{1}$ \\ Rodolfo André Dellagrana ${ }^{2}$ \\ Cassiano Ricardo Rech ${ }^{3}$ \\ Eliane Denise da Silveira Araújo ${ }^{4}$
}

Orlonski S, et al. Estado nutricional e fatores associados ao déficit de estatura em crianças atendidas por uma unidade de ensino básico de tempo integral. Rev Bras Crescimento Desenvolvimento Hum. 2009; 19(1): 54-62.

\section{Resumo:}

O objetivo é analisar o estado nutricional e os fatores associados ao déficit estatural em crianças de quatro a 10 anos de idade. Trata-se de um estudo transversal que avaliou 335 escolares atendidos por uma Unidade de Ensino Básico de tempo Integral da cidade de Ponta Grossa, no ano de 2007. O estado nutricional foi determinado a partir dos índices antropométricos de peso/estatura e estatura/idade. A idade média do grupo foi de 7,7 anos. O déficit de peso/estatura ocorreu em $0,9 \%$ das crianças, a baixa estatura em $6,9 \%$ e o excesso de peso em $27,8 \%$ das crianças. A análise univariada demonstrou que a baixa estatura esteve associada às seguintes variáveis: baixo peso ao nascer $\left(\mathrm{RP}=2,59\right.$; $\mathrm{IC}_{95 \%}$ $1,02-4,12)$, baixa escolaridade materna $\left(\mathrm{RP}=2,12 \mathrm{IC}_{95 \%} 1,54-3,18\right)$ e baixo nível sócioeconômico ( $\mathrm{RP}=2,36$; $\mathrm{IC}_{95 \%}$ 2,26-4,46). As variáveis sexo, idade, número de irmãos e número de pessoas no domicílio não estiveram associadas com a baixa estatura $(\mathrm{p}<0,05)$. Assim, concluiu-se que houve uma elevada prevalência de excesso de peso na amostra, a taxa de baixa estatura é semelhante à apresentada em outros estudos e a baixa estatura se mostrou associada à condição econômica da família, peso ao nascer e escolaridade materna.

Palavras-chave: estado nutricional; antropometria; criança; excesso de peso; déficit de crescimento.

1 Acadêmica do Curso de Educação Física, Universidade Estadual de Ponta Grossa, Paraná, Brasil.

2 Professor de Educação Física, Mestrando do Programa de Pós-Graduação em Educação Física, Universidade Federal do Paraná, Curitiba, Brasil.

3 Mestre. Professor Assistente, Departamento de Educação Física, Universidade Estadual de Ponta Grossa, Paraná, Brasil.

4 Mestre. Professora Colaboradora, Departamento de Educação Física, Universidade Estadual de Ponta Grossa, Paraná, Brasil. Correspondência para: Cassiano Ricardo Rech. Dezenove de Dezembro, 344 apt 102, Centro - Ponta Grossa, Paraná, Brasil. CEP: 84010 - 390. Fone: (42- 3225 5464).

E-mail: crrech@hotmail.com 


\begin{abstract}
:
The aim of the present study is to analyze the nutrition status and the associated factors of low stature in children between four and ten years old. It is a transversal study that evaluated 335 students attended by a regular school of integral time of Ponta Grossa, in 2008. The nutritional status was determinated through weight/height and height/age indices. The medium age is 7.7 years old. $0.9 \%$ of the children presented malnourishment, $6.9 \%$ presented low stature and $27.8 \%$ presented overweight. The analysis was making with just one variation and demonstrated that the low stature is related to the variables: low weight when children were born ( $\mathrm{RP}=2.59$, IC95\% 1.02 - 4.12), mothers with a little education level $(\mathrm{RP}=2.12$ IC95\% 1.54 - 3.18), low economical and social level (RP= 2. 36, IC95\% 2.26 - 4.46). The variables sex, age, number of brothers and number of people at home weren't associated with the low stature $(\mathrm{p}<0.05)$. This study concluded that happened a big overweight prevalence, the condition, weight when children were born and mothers educational level.
\end{abstract}

Key words: nutritional status; anthropometry; children; overweight; growth insufficiency.

\section{INTRODUÇÃO}

O crescimento físico é um processo dinâmico e contínuo que ocorre desde a concepção até o final da vida, expresso pelo aumento do tamanho corporal, apresenta especificidades em cada etapa da vida e sofre influência de vários fatores, tanto genéticos como ambientais. ${ }^{1}$ Deste modo, a avaliação do crescimento é um importante instrumento para se conhecer a saúde, tanto individualmente, quanto coletivamente ${ }^{2}$, assim como para detectar e corrigir problemas com repercussões futuras para a saúde, como obesidade, doenças crônicas, baixa estatura, entre outros.

Considerando que todo o ser humano nasce com um potencial genético de crescimento que poderá ou não ser alcançado dependendo das condições de vida a que esteja exposto desde a concepção até a idade adulta, é necessário investigar a influencia dos fatores intrínsecos (genéticos) e extrínsecos (ambientais) no crescimento físico. ${ }^{3}$

Dentre os fatores extrínsecos, a situação socioeconômica, cultural, permeiam o crescimento infantil desde a fase pré-natal, podendo afetar o quadro nutricional da mãe durante a gravidez, e consequentemente retardo de crescimento intra-uterino e agravo interrelacionado com a prematuridade e com o baixo peso ao nascer e se constituem em importantes fatores intermediários do crescimento posterior da criança merecendo, portanto, destaque ao se avaliar o crescimento infantil. ${ }^{1-3}$

A prematuridade, independentemente das características econômicas e sociais da família, implica em risco nutricional maior ao longo da infância, com todas as desvantagens que isso representa para o desenvolvimento do indivíduo. ${ }^{1,4}$

Outro aspecto importante ligado ao crescimento físico e mudanças no estado nutricional de crianças refere-se ao aumento da prevalência de sobrepeso e obesidade na população infantil no Brasil ${ }^{4,5}$ e no mundo. ${ }^{6}$

Assim como a desnutrição esteve sempre ligada à menor renda, a obesidade esteve associada à abundância, sendo o sujeito obeso considerado como rico e saudável e em outros tempos também como padrão de beleza. $\mathrm{E}$ a idéia de que gordo é saudável muitas vezes é considerada como sinônimo de alguém que se curou de doença grave. ${ }^{2}$ Portanto, além de identificar os casos de obesidade e déficit de 
crescimento é importante identificar os fatores que proporcionam à criança um índice de crescimento menor do que o esperado.

Portanto, avaliações em diferentes etapas da vida do ser humano em crescimento, assim como o acompanhamento do crescimento, podem contribuir para explicar fatos que ocorreram em fases anteriores e sugerir riscos imediatos, mediatos e tardios para a saúde de crianças.

Com isso, o presente estudo tem como objetivo diagnosticar o estado nutricional e verificar os fatores associados ao déficit de estatura em crianças atendidas por uma Unidade de Ensino Básico de tempo integral da cidade de Ponta Grossa, Paraná.

\section{MÉTODO}

O presente estudo caracteriza-se como um estudo descritivo de associação e de corte transversal, composto por crianças de quatro a 10 anos de idade, matriculadas no ensino básico do Centro de Atenção à Criança e ao Adolescente (CAIC) do Município de Ponta Grossa, Paraná. A população foram todas as 386 crianças de quatro a 10 anos de idade devidamente matriculadas no CAIC. A amostra, devido ao número reduzido de crianças, foi toda a população, com exceção das 51 crianças cujos pais não assinaram o termo de consentimento livre e esclarecido, o questionário ou que não compareceram no dia da avaliação, totalizando um número de 335 alunos (173 meninas e 162 meninos).

Os procedimentos adotados durante a realização do estudo foram aprovados pelo Comitê de Ética em Pesquisa da Universidade Estadual de Ponta Grossa, Paraná.

Foram tomadas as medidas de altura e peso corporal, além das informações do nível socioeconômico (NSE), peso da criança ao nascer, idade da mãe, escolaridade materna, número de irmãos e número de pessoas na residência. Estas informações foram obtidas através de um questionário entregue aos pais ou responsável antes da data da coleta.

Para a classificação do NSE foi utilizada a padronização da Associação Nacional de Empresas de Pesquisa. ${ }^{7}$ Foram realizados agrupamentos das classes A1, A2 e $\mathrm{A} 3$, para um único grupo $(\mathrm{A})$, o mesmo aconteceu para as classes B1 e B2 agrupadas em B, e ainda, C e D.

O peso corporal foi medido utilizando uma balança da marca Filizola ${ }^{\circledR}$ com escalas de 100 gramas, e a estatura foi mensurada utilizando o estadiômetro fixado à balança, com precisão de 0,1 cm. Em ambas as medidas as crianças permaneciam descalças e com o uniforme escolar. A partir destas mensurações foi calculado o Índice de Massa Corpórea $\left(\mathrm{kg} / \mathrm{m}^{2}\right)$. Estas medidas foram coletadas em uma sala reservada na própria escola no horário das aulas de Educação Física.

O estado nutricional das crianças foi classificado pelo índice de estatura/idade (E/I) e peso/estatura (P/E), expressado em valor de escore $\mathrm{Z}$ em relação à mediana da população de referência ${ }^{8}$, recomendado pela Organização Mundial da Saúde ${ }^{9}$. Sendo que um escore $\mathrm{Z} \leq 2$ foi caracterizado como baixo peso e baixa estatura. Já escore $Z \geq 2$ foi caracterizado como excesso de peso para a relação peso/ estatura.

Para o tratamento estatístico utilizouse inicialmente de estatística descritiva, agrupando os resultados em valores de média e desvio-padrão. O teste $t$ não pareado foi utilizado para comparação de médias entre os sexos. Para verificar a associação entre déficit estatural e as variáveis de sexo, peso ao nascer, nível socioeconômico, idade da mãe, número de irmãos e número de pessoas na residência foram calculadas a razão de prevalência (RP) com seu respectivo intervalo de confiança (IC) de 95\%. A análise dos dados foi realizada no software SPSS, versão 11.0 e foi adotado nível de significância de $\mathrm{p}<0,05$. 
Tabela 1: Variáveis descritivas de crianças atendidas por uma Unidade de Ensino Básico de Tempo Integral, Ponta Grossa, 2007.

\begin{tabular}{lccccccc}
\hline \multirow{2}{*}{ Variáveis } & \multicolumn{2}{c}{ Meninos } & \multicolumn{2}{c}{ Meninas } & \multicolumn{2}{c}{ Total } & \multirow{2}{*}{$\mathbf{P}^{*}$} \\
& média & $\mathrm{dp}$ & média & $\mathrm{dp}$ & média & $\mathrm{dp}$ & \\
\hline Idade (anos) & 7,7 & 1,71 & 7,7 & 1,74 & 7,7 & 1,72 & \\
Peso Corporal $(\mathrm{kg})$ & 26,4 & 6,74 & 27,6 & 8,73 & 27,1 & 7,84 & \\
Estatura $(\mathrm{cm})$ & 123,1 & 11,4 & 125,3 & 11,7 & 124,2 & 11,6 & $\mathrm{p}>0,05$ \\
IMC $\left(\mathrm{kg} / \mathrm{m}^{2}\right)$ & 17,3 & 2,16 & 17,4 & 3,06 & 17,3 & 2,66 & \\
\hline
\end{tabular}

* teste t não pareado.

\section{RESULTADOS}

Para as variáveis descritivas de idade, peso corporal, estatura e índice de massa cor- poral (IMC) não foram encontradas diferenças significativas entre os sexos (tabela 1 ). O estado nutricional baseado no índice de peso/estatura é apresentado na tabela 2.

Tabela 2: Análise do estado nutricional das crianças atendidas por uma Unidade de Ensino Básico de Tempo Integral, Ponta Grossa, 2007.

\begin{tabular}{lrrrrrr}
\hline \multirow{2}{*}{ Variáveis } & \multicolumn{2}{c}{ Meninos } & \multicolumn{2}{c}{ Meninas } & \multicolumn{2}{c}{ Total } \\
& $n$ & $\%$ & $n$ & $\%$ & $n$ & $\%$ \\
\hline Desnutrição & - & - & 3 & 1,7 & 3 & 0,9 \\
Eutrófico & 126 & 77,8 & 113 & 65,3 & 239 & 71,3 \\
Excesso peso & 36 & 22,2 & 57 & 33,0 & 93 & 27,8 \\
\hline
\end{tabular}

Observou-se uma prevalência de $27,8 \%$ de excesso de peso, sendo 22,2\% entre os meninos e $33 \%$ nas meninas. A tabela 3 apresenta a prevalência de déficit de estatura (DE) para o indicador de estatura/idade.

Tabela 3: Análise do estado nutricional das crianças analisadas no estudo para o indicador de estatura / idade.

\begin{tabular}{lrrrrrr}
\hline \multirow{2}{*}{ Variáveis } & \multicolumn{2}{c}{ Meninos } & \multicolumn{2}{c}{ Meninas } & \multicolumn{2}{c}{ Total } \\
& \multicolumn{1}{c}{$n$} & \multicolumn{1}{c}{$\%$} & \multicolumn{1}{c}{$n$} & \multicolumn{1}{c}{$\%$} & \multicolumn{1}{c}{$\%$} \\
\hline Baixa estatura & 15 & 9,3 & 8 & 4,6 & 23 & 6,9 \\
Estatura normal & 147 & 92,6 & 165 & 96,5 & 312 & 93,1 \\
\hline
\end{tabular}

Nota-se que a prevalência de desnutrição por baixa estatura foi de 6,9\% (9,3\% meninos e $4,6 \%$ meninas), com RP de 2,10 para o sexo masculino (tabela 4). A tabela 4 apresenta outras variáveis associadas à baixa estatura sendo que foi utilizada a análise da razão de prevalência (RP). 
Tabela 4: Fatores associados à baixa estatura em crianças de seis a dez anos de idade atendidos por uma Unidade de Ensino Básico de Tempo Integral, Ponta Grossa, 2007.

\begin{tabular}{|c|c|c|c|c|}
\hline Variáveis & $\mathbf{n}$ & $\%$ & $\mathbf{R P}\left(\mathbf{I C}_{95 \%}\right)$ & $\mathbf{p}^{*}$ \\
\hline \multicolumn{5}{|l|}{ Sexo } \\
\hline Masculino & 15 & 9,3 & $2,10(0,87-5,36)$ & \\
\hline Feminino & 8 & 4,6 & 1 & \\
\hline Peso ao nascer* & & & & $0,001 *$ \\
\hline$<2500 \mathrm{~g}$ & 18 & 21,8 & $2,59(1,02-4,12)$ & \\
\hline$>2500 \mathrm{~g}$ & 5 & 2,0 & 1 & \\
\hline Escolaridade materna* & & & & $0,001 *$ \\
\hline$<4$ anos & 12 & 10,4 & $2,12(1,54-3,18)$ & \\
\hline $5-8$ anos & 6 & 5,4 & $1,98(0,86-6,05)$ & \\
\hline$>9$ anos & 5 & 4,5 & 1 & \\
\hline Classe econômica* & & & & $0,001 *$ \\
\hline A & 5 & 5,0 & 1 & \\
\hline B & 7 & 6,1 & $1,25(1,14-5,15)$ & \\
\hline C e D & 11 & 8,8 & $2,36(2,26-4,69)$ & \\
\hline
\end{tabular}

IC95\% = intervalo de confiança 95\%; RP = razão de prevalência.

* significativo estatisticamente para $\mathrm{p}<0,05$.

Observou-se que as variáveis de sexo, número de irmãos e número de pessoas no domicílio não estiveram associadas à baixa estatura $(\mathrm{p}<0,05)$.

Houve associação positiva entre a baixa estatura e o baixo peso ao nascer ( $\mathrm{RP}=2,59$; $\left.\mathrm{IC}_{95 \%}=1,02-4,12\right)$. Observou-se que 21,8\% das crianças que apresentavam baixo peso ao nascer tem um risco 2,59 vezes maior de apresentar baixa estatura na infância do que aqueles que não apresentaram baixo peso ao nascer.

Outra variável que se mostrou associada à baixa estatura (tabela 4) foi a baixa escolaridade da mãe $\left(\mathrm{RP}=2,12\right.$; $\mathrm{IC}={ }_{95 \%} 1,54-$ $3,18)$. Nota-se que mães com menos de 4 anos de estudo apresentam um risco 2,12 vezes maior de possuir um filho com baixa estatura do que uma mãe que possui mais de 9 anos de estudo.

Além da escolaridade da mãe, observouse que o nível sócio-econômico familiar é associado com a baixa estatura na amostra. Sendo que a prevalência de baixa estatura está diretamente relacionada com a classe econômica. A prevalência de baixa estatura foi $5 \%$ na classe econômica $A$, já para a classe C e D esta prevalência foi de 8,8\%.

A análise univariada mostra que filhos nascidos em famílias de classes econômicas $C$ e D apresentam um risco 2,36 vezes maior de desenvolver baixa estatura em comparação com as famílias de nível socioeconômico A e B. 


\section{DISCUSSÃO}

Evidências científicas apontam para um aumento nos casos de sobrepeso e obesidade em crianças e adolescentes em vários países. ${ }^{10,11}$ No Brasil, a situação não é diferente ${ }^{5,12}$, refletindo uma transição nutricional pela qual o país está passando e que exige intervenções nutricionais adequadas. ${ }^{5,13}$ Este fato ganha relevância, pois indivíduos obesos na infância apresentam elevado risco de permanecerem obesos na vida adulta. ${ }^{14}$

Os resultados do estudo apontam prevalência de excesso de peso de 27,8\% na amostra analisada, sendo maior em relação ao estudo de Salomons et al. ${ }^{15}$ na cidade de ArapotiPR, com crianças da rede Municipal de Ensino, que encontraram uma prevalência de 20,9\% de excesso de peso. E ainda, apresentando valores maiores em relação aos de Abrantes et al. ${ }^{4}$, em crianças das regiões Sudeste e Nordeste, possivelmente pelo fato de que a região Sul do país apresenta maior prevalência de obesidade, sendo semelhantes e até mesmo superiores às de países desenvolvidos. ${ }^{16}$

O quadro de desnutrição infantil tem diminuído, sendo que no Brasil a prevalência de crianças desnutridas apresenta decréscimo acentuado nos últimos anos, porém, a desnutrição ainda é considerada um problema de saúde pública em países em desenvolvimento. ${ }^{17}$

A análise do estado nutricional de crianças é de fundamental importância, principalmente no que se refere à desnutrição, pois esta pode comprometer de maneira rigorosa o crescimento linear da criança. ${ }^{18}$ Ela é causada pela falta de alimentação e como efeito de doenças, estando ainda relacionada com mortalidade prematura. $^{9}$
Foi observada uma prevalência de 0,9\% de desnutrição aguda (peso/estatura), valor este que corrobora com o estudo de Zollner e Fisberg ${ }^{19}$ ao descrever o estado nutricional de crianças assistidas em creches do município de São Paulo. Entretanto é menor que os valores relatados por Salomons et al. ${ }^{15}$ e Biscegli et al. ${ }^{20}$, que encontraram valores de desnutrição para o indicador de peso/estatura de 10,1\% e $11,5 \%$, respectivamente. Possivelmente a prevalência foi pequena, pois o Centro de Atenção à Criança e ao Adolescente (CAIC) é uma escola de período integral, onde as crianças realizam a maioria de suas refeições diárias, assim como em creches públicas, obtendo uma alimentação balanceada oferecendo suporte nutricional, durante a semana de aula.

Os valores de desnutrição pregressa (estatura/idade) foram mais elevados (6,9\%), semelhantes aos valores encontrados por Neves et al. ${ }^{21}$ em crianças da cidade de Belém, Pará, que foram de 7,8\%. Entretanto, menores do que os resultados encontrados por Laurentino et al. ${ }^{22}$ em crianças pernambucanas, com prevalência de 16,9\% de retardo estatural pelo indicador de estatura/idade, e por Farias e Petroski ${ }^{23}$ que apresentaram valores bem menores em relação a crianças de Porto Velho, Rondônia, com 27,2\% de baixa estatura.

A partir de uma amostra representativa de crianças brasileiras Engstrom e Anjos ${ }^{24}$ mostraram prevalências de $14,4 \%$ de déficit estatural, sendo que, as regiões Norte e Nordeste do país são as que apresentam maiores percentuais de desnutrição pelo indicador de estatura/idade, com $21,5 \%$ e $24,9 \%$, respectivamente, deste modo podendo explicar as altas prevalências de baixa estatura dos estudos citados em relação à presente investigação. 
Outro fator considerado de risco para a mortalidade infantil é o baixo peso ao nascer ( $<2500 \mathrm{~g}$ ), pois pode causar desordens no desenvolvimento da criança. ${ }^{25} \mathrm{Na}$ investigação conduzida por Gigante et al. ${ }^{5}$ na cidade de Pelotas, Rio Grande do Sul, crianças que nasceram com baixo peso ao nascer apresentaram maior incidência de déficit de crescimento em relação às crianças que nasceram com peso $>$ 2500g. Estes resultados são semelhantes aos encontrados no presente estudo, onde se observou uma associação entre o baixo peso ao nascer e a baixa estatura na infância ( $\mathrm{RP}=2,59$; IC95\% $=1,02-4,12$ ).

A desnutrição devida à baixa estatura também tem se mostrado associada predominantemente com a escolaridade dos pais e com as condições sócio-econômicas da família. ${ }^{26}$ No que se relaciona com a escolaridade materna, é evidenciado que crianças cujas mães têm escolaridade inferior a quatro anos apresentam um risco maior para baixa estatura, apresentando decréscimo conforme a escolaridade da mãe aumenta, fato este que provavelmente está relacionado com a importância que cuidados apropriados têm para a saúde e nutrição da criança, pois mães que apresentam uma escolaridade menor na maioria dos casos têm menor acesso à educação, á saúde e condições básicas de vida, dando assim uma menor atenção aos filhos.

Os resultados encontrados neste estudo estão de acordo com outras investigações, que demonstram relação inversa entre a prevalência de baixa estatura e a escolaridade materna. ${ }^{21,24,27}$

Neste sentido, Poel et al. ${ }^{28}$ relatam que além de preocupações com a desigualdade socioeconômica, são de fundamental importân- cia preocupações fixando políticas de saúde. $\mathrm{O}$ presente estudo diagnosticou que quanto ao NSE familiar, as crianças pertencentes às classes econômicas $\mathrm{C}$ e D apresentam risco maior para baixa estatura $(\mathrm{RP}=2,36$; IC95\%=2,264,69 ), sendo este risco menor para crianças que se encontram nas classes $B(R P=1,25$; IC95\%=1,14-5,15). Estes achados corroboram com outros estudos que indicam que crianças em condições socioeconômicas mais favoráveis apresentam um menor risco de apresentar baixa estatura. ${ }^{19,29}$

Deste modo, fica evidente que mesmo em uma escola de tempo integral, na qual são realizadas as maiorias das refeições, os aspectos sócio-demográficos, econômicos da família e de condições de vida das crianças estão diretamente relacionadas com os casos de baixa estatura.

Assim, houve uma elevada prevalência de excesso de peso e baixa estatura na amostra, entretanto notou-se uma pequena prevalência de baixo peso. Observou-se que as variáveis de baixo peso ao nascer, baixa escolaridade da mãe e baixo nível econômico familiar estão associados com a baixa estatura em crianças.

Devido aos elevados índices de excesso de peso recomenda-se um trabalho de intervenção nutricional e de atividades física na escola. Com relação à baixa estatura, fica evidente que as políticas públicas devem atender não só os núcleos comuns (escolas), mas também, principalmente, as famílias em seu ambiente de vida diária, tanto por meio de aumento da escolaridade das mães, quanto por um maior acesso ao acompanhamento da gravidez e melhora dos níveis econômicos da população. 


\section{REFERÊNCIAS}

1. Spyrides MHC, Struchiner CJ, Barbosa MTS, Kac G. Efeito das práticas alimentares sobre o crescimento infantil. Rev. Bras. Saúde Materna. 2005;5(2):145-153.

2. Zeferino AMB, Barros Filho AA, Bettiol $\mathrm{H}$, Barbieri MA. Acompanhamento do crescimento. J Pediatr. 2003;79(supl.1):23-32.

3. Romani SAM, Lira PIC. Fatores determinantes do crescimento infantil. Rev. Bras. Saúde Matern. Infant. 2004;4(1):15-23.

4. Abrantes MM, Lamounier JA, Colosimo EA. Prevalência de sobrepeso e obesidade em crianças e adolescentes das regiões Sudeste e Nordeste. J Pediatr. 2002;78(4):335-340.

5. Gigante DP, Victora CG, Araújo CLP, Barros FC. Tendências no perfil nutricional das crianças nascidas em 1993 em Pelotas, Rio Grande do Sul, Brasil: análises longitudinais. Cad. Saúde Pública. 2003;19(supl.1):S141S147.

6. Wang Y, Monteiro CA, Popkin BN. Trends and obesity and underweight in older children and adolescents in the United States, Brazil, China, and Russia. Am J Clin Nutr. 2002;75(6):971-977.

7. Associação Brasileira de Empresas de Pesquisa - ABEP. Critério de Classificação Econômica Brasil. 2003. Acessado em 18 de outubro de 2008. Disponível em <http: //www.abep.org>.

8. CDC (Center for Disease Control and Prevention). 2000 CDC Growth charts:
United States [Online] Hyaltsville: 2002. Acessado em 14 de outubro de 2008. Disponível em: http://www.cdc.gov/ growthcharts.

9. World Health Organization. Physical Status: The Use and Interpretation of Anthropometry. Report of a WHO Expert Committee. 1995. Technical Report Series No. 854. Geneva: WHO.

10. Tremblay MS, Willms JD. Secular trends in the body mass index of Canadian children. CMAJ. 2000;163(11):1429-1433.

11. Bundred P, Kitchiner D, Buchan I. Prevalence of overweight and obese children between 1989 and 1998: population based series of cross sectional studies. BMJ. 2001;322(7282):326-328.

12. Veiga GV, Cunha AS, Sichieri R. Trends in overweight among adolescents living in the poorest and richest regions of Brazil. Am J Public Health. 2004;94(9):1544-1548.

13. Batista Filho M, Rissin A. A transição nutricional do Brasil: tendências regionais e temporais. Cad. Saúde Pública. 2003;19(supl 1):S181-S191.

14. Whitaker RC, Wright JA, Pepe MS, Seidel KD, Dietz WH. Predicting Obesity in Young Adulthood from Childhood and Parental Obesity. N Engl J Med. 1997;337(13):869-873.

15. Salomons E, Rech CR, Loch MR. Estado nutricional de escolares de seis a dez anos de idade da rede municipal de ensino de Arapoti, Paraná. Rev. Bras. Cineantropom. Desempenho Hum. 2007;9(3):244-249. 
16. Gigante DP, Barros FC, Post CLA, Olinto MTA. Prevalência de obesidade em adultos e seus fatores de risco. Rev. Saúde Pública. 1997;31(3):236-246.

17. Onis M, Frongillo EA, Blössner M. Is malnutrition declining? An analysis of changes in levels of child malnutrition since 1980. Bulletin of the World Health Organization. 2000;78(10):1222-1233.

18. Monteiro CA, Conde WL. Tendência secular da desnutrição e da obesidade na infância na cidade de São Paulo (19741996). Rev. Saúde Pública. 2000;34(supl.6):52-61.

19. Zollner CC, Fisberg RM. Estado nutricional e sua relação com fatores biológicos, sociais e demográficos de crianças assistidas em creches da prefeitura do município de São Paulo. Rev. Bras. Saúde Matern. Infant. 2006;6(3):319-328.

20. Biscegli TS, Polis LB, Santos LM, Vicentin M. Avaliação do estado nutricional e do desenvolvimento neuropsicomotor em crianças freqüentadoras de creche. Rev Paul Pediatr. 2007;25(4):337-342.

21. Neves OMD, Brasil ALD, Brasil LMBF, Taddei JAAC. Antropometria de escolares ao ingresso no ensino fundamental na cidade de Belém, Pará, 2001. Rev. Bras. Saúde Matern. Infant. 2006;6(1):39-46.

22. Laurentino GEC, Arruda IKG, Raposo MCF, Batista Filho M. Déficit estatural em crianças em idade escolar e em menores de cinco anos: uma análise comparativa. Rev. Nutr. 2006;19(2):157167.
23. Farias ES, Petroski EL. Estado nutricional e atividade física de escolares da cidade de Porto Velho, RO. Rev. Bras. Cineantropom. Desempenho Hum. 2003;5(1):27-38.

24. Engstrom EM, Anjos LA. Déficit estatural nas crianças brasileiras: relação com condições sócio-ambientais e estado nutricional materno. Cad. Saúde Pública. 1999;15(3):559-567.

25. Thompson LA, Goodman DC, Chang $\mathrm{CH}$, Stukel TA. Regional Variation in Rates of Low Birth Weight. Pediatrics. 2005;116(5):1114-1121.

26. Victora CG. The association between wasting and stunting: An international perspective. J Nutr. 1992;122(5):11051110.

27. Olinto MTA, Victora CG, Barros FC, Tomasi E. Determinantes da Desnutrição Infantil em uma População de Baixa Renda: um Modelo de análise Hierarquizado. Cad. Saúde Pública. 1993;9(supl.1):14-27.

28. Poel EV, Hosseinpoor AR, Speybroeck N, Ourti TV, Vega J. Socioeconomic inequality in malnutrition in developing countries. Bulletin of the World Health Organization. 2008;86(4):282-291.

29. Fisberg RM, Marchioni DML, Cardoso MRA. Estado nutricional e fatores associados ao déficit de crescimento de crianças freqüentadoras de creches públicas do Município de São Paulo, Brasil. Cad. Saúde Pública. 2004;20(3):812-817.

Recebido em: 17/11/2008 Modificado em: 20/01/2009 Aceito em: 02/02/2009 